\title{
Approfondir la Méthode de François Delsarte : Les attitudes des jambes
}

\author{
Franck Waille \\ Laboratoire de Recherche Historique Rhône-Alpes/LARHRA, France \\ E-mail: franck.cw@gmail.com
}

Résumé

La reconstruction des enseignements pratiques (pour le corps, et encore plus la voix) de François Delsarte relève d'un vrai défit méthodologique : si ces enseignements ont joué un rôle déterminant dans l'émergence de la modernité des arts de la scène, des pratiques psychosomatiques et de l'éducation somatique, ils ont essentiellement été transmis de manière " orale ", de maître à élève. Et ils ont été, pour une part, perdus. C'est à partir des éléments de transmission textuelle, associés à une pratique de la danse moderne, du théâtre et des approches somatiques, qu'il est possible d'en retrouver une compréhension aussi proche que possible de leurs versions d'origine, sans avoir la prétention de pouvoir revenir à celles-ci. D'autant plus que les très nombreuses nuances que Delsarte introduisait dans son enseignement invitent à une dynamique ouverte : non chercher « la lettre » mais «l'esprit » de son travail, dans une perspective de renouvellement constant. Cette démarche est celle de la recherche de Franck Waille, qui ne cesse de s'affiner depuis le début des années 2000. Elle concerne différents éléments de la Méthode somatique expressive de Delsarte. Cet article présente une mise à jour concernant les attitudes des jambes, favorisée par la rencontre avec la recherche de Clóvis Massa sur Delsarte faite au début des années 1990.

Mots-clés

François Delsarte. Attitudes / Accord de neuvième. Expression des jambes. Arts de la scène. Éducation somatique.
Abstract

The reconstruction of the practical teachings (for the body, and even more the voice) of François Delsarte raises a real methodological challenge: if these teachings played a determining role in the emergence of the modernity of the performing arts, practices psychosomatic and somatic education, they were essentially transmitted "in an oral way" from master to student. And they were, for one, lost. It is from the elements of textual transmission, associated with a practice of modern dance, drama and somatic approaches, that it is possible to find an understanding as close as possible to their original versions, without pretending to be able to return to these. Especially since the many nuances that Delsarte introduced into his teaching invite an open dynamic: not looking for "the letter" but "the spirit" of his work, in a perspective of constant renewal. This approach is that of the research of Franck Waille, which has been constantly refined since the beginning of the 2000s. It concerns different elements of Delsarte's Expressive Somatic Method. This article presents an update on the attitudes of the legs, favored by the meeting with the research of Clóvis Massa on Delsarte made in the early 1990 s.

Keywords

François Delsarte. Attitudes / Ninth Agreement. Expression of the legs. Scenic arts. Somatic education. 


\section{Introduction}

Les enseignements expressifs de François Delsarte (1811-1871), qui ont joué un rôle majeur dans l'émergence de la modernité des arts de la scène en Occident depuis la fin du XIXe siècle, bénéficient depuis les années 1970 d'une série d'études et de publications (universitaires mais pas uniquement) permettant d'en mieux comprendre les contenus et la richesse. Ma propre recherche s'inscrit dans cette dynamique et elle est en renouvellement constant. Ainsi mon ouvrage La méthode somatique expressive de François Delsarte (2016) présentait un approfondissement de certains aspects de ma thèse Corps, arts et spiritualité chez François Delsarte (1811-1871) (2009), en particulier concernant la pédagogie du travail du torse et la compréhension pratique des attitudes des jambes, appelées aussi « attitudes de base » (Cable, s.d., p.1)

Deux périodes ont favorisé la poursuite de l'approfondissement de ma recherche concernant Delsarte. Le postdoctorat fait au Département de Danse de l'Université du Québec À Montréal (UQAM) en 2014 et 2015 m'a permis de poursuivre l'exploration des manuscrits autour du travail du torse. Et c'est lors d'un second postdoctorat au Département d'Éducation de I'Universidade Federal do Rio Grande do Sul (UFRGS) en 2016 et 2017 que j'ai pu revoir une partie de l'approche du travail des attitudes des jambes. Cela a été favorisé par la rencontre avec le travail de Clóvis Massa fait sur Delsarte au début des années 1990. C'est le résultat de ce double approfondissement que je présente ici.

Pour le torse, j'ai pris de la distance vis-àvis des informations fournies par la delsartiste américaine Genevieve Stebbins (1977) qui, si elles ont l'immense avantage d'une très grande clarté (des leçons très organisées et détaillées), présentent des écarts par rapport aux manuscrits plus proches des enseignements originaux de Delsarte. J'ai aussi partiellement revisité l'étude des attitudes des jambes par une relecture des manuscrits.

Le travail des jambes et du torse s'inscrit dans le cadre plus général du travail expressif des différentes parties du corps avec l'outil delsartien appelé l'accord de neuvième. Cet outil a été la source de nombreuses ambiguittés dans la transmission et la réception des enseignements de Delsarte. Aussi, dans un premier temps, je présenterai une synthèse concernant l'accord de neuvième, afin d'en bien saisir les dynamiques. Dans un deuxième temps, j'exposerai par des textes et des photographies (qui sont là uniquement pour faciliter la lecture des textes, et qui proviennent de différents séries de prises de vue) les apports combinés de mes recherches et de celles de Clóvis Massa concernant les attitudes des jambes. Enfin, dans un troisième temps (qui fera l'objet d'un article à part ${ }^{1}$ ), je reviendrai sur les attitudes du torse (avec des textes et des images là aussi) et j'élargirai ma présentation par une réflexion sur la notion d'équilibre harmonique caractéristique des transmissions delsartiennes.

\section{Problématique autour des tableaux d'accords de neuvième de Delsarte}

Attitudes et tableaux d'accords de neuvième

Les attitudes du corps sont considérées par

1 Approfondir la Méthode de François Delsarte : Les attitudes du torse et l'equilibre harmonique. 
Delsarte comme la base de la dynamique. Leur travail est conçu dans le cadre des tableaux d'accords de neuvième, qui présentent un cadre résumant son système théorico-théologique. Travailler les attitudes peut être considéré comme la troisième étape du travail somatique expressif, après celle de la prise de conscience de sa propre verticalité, puis celle de la décontraction (décomposition) des différentes articulations (cf. Waille, 2016, chapitre 7).

Les attitudes sont les dispositions au mouvement, comme les sentiments sont pour Delsarte les dispositions à l'acte (cf. Waille, 2011, p. 79-93). C'est en ce sens qu'il est possible de comprendre que « l'attitude est le mouvement intrinsèque » (Mackaye, 1869/1870b, p. 129), la manifestation la plus immédiate d'un mouvement intérieur, c'est-à-dire d'un sentiment. Le terme de mouvement pour parler des attitudes projette celles-ci dans une perspective dynamique, ce qui trouve un écho dans les recherches en physiologie du mouvement : Alain Berthoz indique, après avoir présenté la théorie de la synergie du physiologiste Nicolaï Aleksandrovitch Bernstein (1896-1966), que « le mouvement ne serait donc qu'un glissement progressif de postures » (Berthoz, 1997, p. 24). Cela trouve également un écho dans deux approches contemporaines du mouvement liées à l'éducation somatique. Hubert Godard (1995) prend en compte dans son analyse du mouvement le fond (qui correspond ici à l'attitude), et la figure ou le geste. Warren Lamb, collaborateur de Rudolf Laban, envisage lui la fusion de la posture et du geste (Posture Gesture Mergers - PGMs) : le geste est soutenu par la posture, la posture soutient la qualité du geste (cf. Fernandes, 2014, p. 137-138). Autrement dit, l'attitude correspondrait au fond pour Godard et à la base de la qualité de la forme pour Lamb et Laban. Delsarte analyse le mouvement expressif comme étant ancré dans la posture, dans les attitudes, bases de la dynamique.

Les attitudes sont donc pour lui les positions multiples que peuvent prendre les différentes parties du corps en lien avec différents sentiments ou mouvements intérieurs. Leur aspect permanent indique que le corps, qu'il soit immobile ou en mouvement, est toujours lié, dans sa manière d'être, à un état intérieur. À nouveau, des liens sont possibles en direction des neurosciences où existe la notion de « répertoire de comportements » (Berthoz, 2003, p. 120).

\section{Dynamiques des tableaux d'accords de neuvième}

Les attitudes ont donné lieu de la part de Delsarte et de ses élèves à de nombreuses représentations graphiques organisées sous la forme d' " accords de neuvième » et concernant toutes les parties du corps. Delsarte tire le terme d' « accord de neuvième » de sa culture musicale, mais il lui donne un sens complètement nouveau et propre à ses enseignements. L'accord de neuvième chez lui est une représentation graphique à but didactique permettant de synthétiser de manière simple une réalité complexe. Aucun des tableaux que Delsarte utilisait durant ses cours ne nous est parvenu car ses documents pédagogiques sont aujourd'hui introuvables (cf. Waille, 2015a). II reste seulement quelques textes issus directement de ses cours ; certains décrivent les attitudes, sans jamais être accompagnés de tableaux.

L'accord de neuvième est basé sur les fondamentaux théoriques des enseignements delsartiens : la notion théologique de circuminces- 
sion (relations entre les différentes personnes trinitaires), associée à la définition thomasienne de la Trinité (cf. Waille, 2011, p. 184225) ; c'est la combinaison des trois « données de base » (en théologie, du Père, du Fils et du Souffle saint), ce qui génère neuf termes. Chacun de ces termes est une combinaison des dynamiques de chacun des trois termes d'origine, que nous pouvons synthétiser ainsi :

* Père : 1, dynamique vitale, mouvement excentrique (du centre vers la périphérie), couleur primaire rouge ;

${ }^{*}$ Fils : 2, dynamique intellective, mouvement concentrique (de la périphérie vers le centre), couleur primaire bleue ;

* Souffle saint : 3 , dynamique animique (« de l'âme ", ou synthétique), mouvement neutre / normal (équilibre des deux précédents), couleur primaire jaune.

L'accord de neuvième général (en partant des couleurs primaires) donnerait ceci (Figure 1):

Figure 1 : Accord de neuvième des couleurs

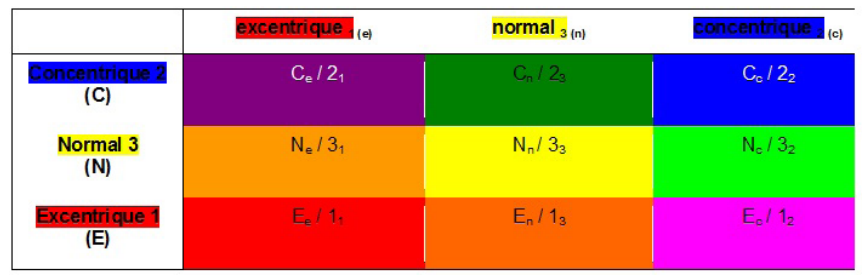

Des indications de type émotionnel accompagnent chaque attitude du corps, comme par exemple sur cet accord de neuvième des attitudes des yeux publié par l'élève de Delsarte, l'abbé Delaumosne (Figure 2):
Figure 2 : Accord de neuvième des attitudes des yeux selon

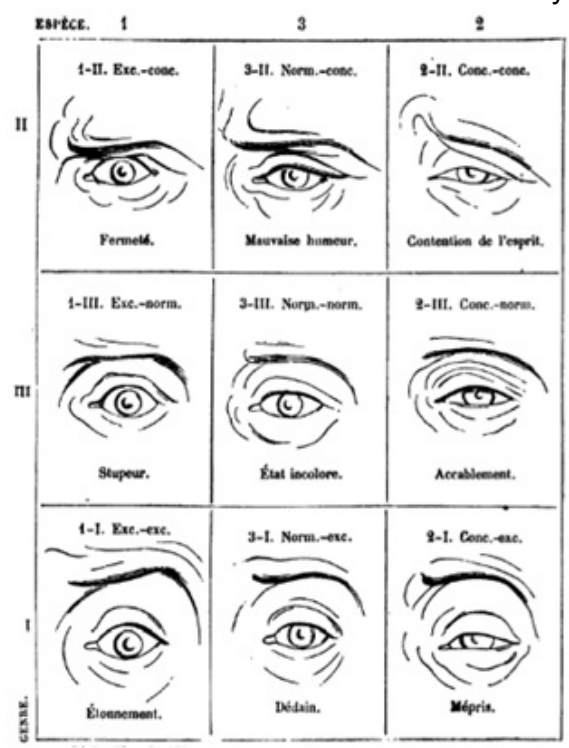

Dans les documents d'archives, ces indications sont toujours plurielles (contrairement à ce qui se trouve dans la publication de Delsaumosne) et pourraient être accompagnées de la mention « etc. » Elles rappellent que les attitudes viennent directement d'observations qui trouvent un cadre d'organisation et de présentation dynamique avec l'accord de neuvième. Delsarte énonce ainse que « ce moyen si simple [lui permet] d'inscrire les faits nombreux qu['il a] poursuivis, et qui s'élèvent à des millions, [sans qu'il se perde] dans ces millions de faits » (Porte, 1992, p. 121). De plus, la mention de ces indications de type émotionnel a une dimension pédagogique : associer un geste à une dimension interne, afin que ce geste soit habité et non mécanique.

Les accords de neuvième sont des outils présentant un processus dynamique qui procède par subdivisions successives : chaque élément, issu du passage de trois genres à neuf espèces, peut être à son tour subdivisé selon la même logique ternaire dans un processus sans fin. Ce processus de démultiplication permet à Delsarte d'affiner son ana- 
lyse pour détailler les phénomènes expressifs, poussant la description de ces phénomènes dans la limite de l'applicable. II dénombre ainsi les combinaisons qu'il avait pu envisager : 729 « physionomies passionnelles d'un même œil » (1859, cours théorique $n^{\circ} 7$ ) sans compter les combinaisons avec les mouvements de la tête, 6561 « mouvements » ou «présentations de la main » (Porte, 1992, p. 142), 42714297 « expressions de la bouche » (Delsarte, 1859, cours théorique $\left.\mathrm{n}^{\circ} 7\right) \ldots$ De plus, il considère les démultiplications des mouvements potentiels par la combinaison des accords de neuvième des différents éléments d'un ensemble, les bras par exemple, ou les combinaisons entre les accords de différentes parties du corps. Avec l'accord de neuvième, il dispose d'une grille de lecture des phénomènes expressifs allant vers l'infini et qu'il ramène à neuf. Non pour réduire tout à neuf, mais pour pouvoir tout travailler à partir de neuf éléments.

Delsarte est ici à la croisée de deux champs de la recherche actuelle en communication : les modèles effecteurs [effector patterns] des émotions (en particulier avec les recherches de Susana Bloch) et la Nouvelle Communication. Les « travaux de Paul Ekman et de Susana Bloch sur le phénomène de feedback psychobiologique dans les émotions physiquement stimulées » (Pradier, 2000, p. 236) tendent à prouver que les phénomènes soustendant une série de mimiques ont un caractère universel. II est possible de constater que devant certaines expressions du visage, des enfants reconnaissent immédiatement telle ou telle volonté expressive (colère etc.) ; c'est sur cela que jouent le mime, le cirque ou les dessins animés. Pour la Nouvelle Communication, il est impossible « d'isoler chaque composant du système de communication global et parler de "langage du corps", "langage des gestes" etc. (...) [car] pas plus que les énoncés du langage verbal, les "messages" issus d'autres modes de communication n'ont de signification intrinsèque : ce n'est que dans le contexte de l'ensemble des modes de communication, lui-même rapporté au contexte de l'interaction, que la signification peut prendre forme »(Winkin, 2000, p. 24). II s'agit donc à la fois de considérer un langage du geste, et ses liens avec les autres langages. Les variantes et les nuances possibles de chaque attitude indiquée dans un tableau d'accord de neuvième tiennent donc à trois choses : la possibilité d'affiner chacune d'elles de manière quasiment illimitée ; les liens avec le contexte de communication créant des interactions avec les autres modes d'expressions (inflexions de la voix, langage articulé) ; les liens avec les différentes parties du corps jouant ensemble dans un même phénomène expressif.

Le caractère archétypal des attitudes chez Delsarte trouve un écho dans la thèse de Marcel Jousse (1925) dans laquelle l'anthropologue a pris comme " hypothèse centrale que toute l'activité de la pensée et du langage peut se résumer à un ensemble de gestes fondamentaux » (Guido, 2007, p. 269), idée également développée par Laban (1997, p. 131, \& 2003, p. 161-162, 179, 204). Leur intégration par un travail répété, comme c'est le cas chez Delsarte, produirait la mise en place de circuits neurologiques permettant une combinaison non limitée pouvant s'adapter à n'importe quelle situation émotionnelle et expressive. Si Delsarte insiste sur la dimension psychoaffective plutôt que sur celle de la pensée (ce que fait Jousse), il y a une parenté forte entre la notion d'attitudes archétypales que nous trouvons chez Delsarte, et celle de mouvements fondamentaux défendue par Jousse. 
Les tableaux d'accord de neuvième des différentes parties du corps forment comme un alphabet corporel dont les éléments peuvent s'agencer selon diverses organisations, tout comme les éléments du langage écrit s'agencent pour former des mots puis des phrases. Nous sommes en présence d'une combinatoire qui n'est, chez Delsarte, pas uniquement descriptive d'une série de mouvements possibles, mais qui est porteuse de sens, c'est-à-dire d'une dimension expressive. Il est significatif que Delsarte ne propose pas ici, pour le travail corporel, de « lexique émotionnel » pour l'ensemble du visage ou pour le corps entier, mais donne des indications spécifiques d'entraînement pour chaque élément expressif corporel. C'est un contresens que d'imaginer ces tableaux d'accords de neuvième comme des répertoires fermés correspondant de manière absolue à telle ou telle émotion (cf. Waille, 2016, chapitre 3).

\section{Ambiguïtés dans les transmissions} du travail des attitudes

II y a des ambiguïtés dans les transmissions faites par les élèves de Delsarte de son travail des attitudes (nous en avons eu un exemple chez Delaumosne avec l'absence de pluralité des émotions associées à une attitude), et des écarts significatifs dans la manière d'en présenter le travail. Ainsi, il n'y pas de tableaux d'accords de neuvième dans les manuscrits de Mackaye mais seulement des textes, très longs car énonçant de très nombreuses combinaisons et perdant la dynamique synthétique (cf. Waille, 2017). D'autres élèves de Delsarte ont publié des tableaux d'accords de neuvième pour les différentes parties du corps (abbé De- laumosne - ses tableaux ont été largement réutilisés en Amérique, en particulier par Stebbins - et Alfred Giraudet), ce qui a à la fois un intérêt pédagogique (les figures remplacent de longues descriptions d'exercices et en facilitent l'accès), mais aussi beaucoup de limites et de dangers potentiels.

En effet, si Delsarte n'a jamais publié et laissé publier ce type de tableaux de son vivant c'est, d'une part, parce que ces tableaux n'ont pas pour fonction première d'exposer d'une doctrine esthétique mais d'être des supports d'entraînement : leur aspect pédagogique et pratique est primordial. Et c'est, d'autre part, sans doute parce qu'il avait pressenti le risque inhérent à toute publication : celui de figer les attitudes dans les mots et les images renvoyant aux émotions.

La publication peut en effet favoriser une lecture erronée de ces tableaux parce qu'elle permet une entrée purement intellectuelle dans leur contenu : les planches peuvent apparaître comme des grilles donnant les différentes « significations émotionnelles » des attitudes présentées, et non comme des exercices utilisables pour développer ses capacités expressives. Cela est favorisé quand il y a absence de pluralité de situations émotionnelles potentielles associées à une attitude, ce qui est en général le cas dans les publications faites après Delsarte. Les planches peuvent donc être «lues » en dehors de tout un contexte sans lequel elles deviennent des caricatures. Les publications de tableaux d'accords de neuvième ont joué un rôle évident dans les déformations et les mésinterprétations qui ont parfois entouré les enseignements de Delsarte (cf. Waille, 2015b).

II convient donc de concevoir ces tableaux d'abord comme une série de gammes corporelles : « la succession des mouvements d'un 
même agent peut se faire presque de toutes les manières, comme une suite de notes peut s'enchaîner dans une mélodie » (Giraudet, 1895, p. 119). Les neuf cases de chaque tableau sont autant de «notes » qui doivent pouvoir s'enchaîner les unes aux autres et permettre aux yeux, à la bouche, aux bras... d'acquérir une aisance expressive, tout comme les doigts du pianiste se délient en s'exerçant aux différentes gammes musicales. Les tableaux sont des séries d'exercices précis pour chacune des parties du corps, et tous les tableaux d'accords de neuvième peuvent être vus comme autant de gammes corporelles expressives formant un recueil d'entraînement pour l'artiste.

C'est en ayant à l'esprit ces dynamiques et ces nuances qu'il est possible d'aborder le travail des jambes et du torse dans le cadre de l'accord de neuvième.

\section{Les attitudes des jambes : apports combinés des recherches de Clóvis Massa (1991) et de Franck Waille (2009 et 2016)}

Je présente ici une approche renouvelée du travail des attitudes des jambes chez Delsarte. II se trouve que ces attitudes sont aujourd'hui celle qui sont les mieux documentées (cf. Waille, 2011, p. 763-783). J'ai par le passé proposé deux séries de photographies de ces attitudes, basées sur mes interprétations de manuscrits d'élèves de Delsarte ou de Delsarte lui-même, et de dessins de ce dernier. J'ai fait une première lecture de ces documents dans ma thèse (2009), lecture que j'ai affinée pour les actes du colloque « François Delsarte. Mémoire et héritages » (cf. Waille, 2015d) (informations reprises dans Waille, 2016, p. 339-365).
J'amène ici des éléments nouveaux concernant l'approche de ces attitudes, qui viennent en particulier de mes échanges avec Clóvis Massa et de la rencontre avec sa recherche sur Delsarte faite dans les années 1990. Cette recherche, effectuée par un acteur à la fin de sa formation en Licence en Arts de la Scène au Département d'Art Dramatique de I'Université Fédérale du Rio Grande do Sul, alliait intégralement la théorie et la pratique. Depuis Janis Dawn Clarke (1982), c'est (sans doute) le premier travail concernant Delsarte associant analyse théorique et pratique expressive.

Le projet de Graduação (Licence) de Clóvis Massa, défendu en 1991, portait sur l'emploi de répertoires de signes gestuels au théâtre. Massa a utilisé des éléments de la méthode delsartienne comme entrainement physique et comme développement technique dans le processus de mise en scène des pièces Le chant du cygne (Tchekov) et Língua de Trapo (inspirée de Grouxo Marx). Ce projet s'est concrétisé par les mises en scène de ces deux pièces et par une démonstration devant un jury (tout cela a été filmé), ainsi que par le rapport $A$ significação corporal a partir de François Delsarte [La signification corporelle selon François Delsarte].

Clóvis Massa a utilisé comme source principale l'ouvrage de Stebbins (1977), qui présente une synthèse de la transmission faite par Mackaye, des livres d'élèves de Delsarte traduits en anglais et de la rencontre de l'auteure avec des élèves de Delsarte à Paris (cf. Waille, 2015c). II est notable que Massa en soit arrivé à inventer un système d'écriture de l'enchainement des différentes attitudes, système d'écriture inexistant dans la littérature qu'il a consulté mais qui a de très fortes parentés avec une proposition de notation exposée par l'élève de Delsarte Alfred Giraudet (1895). Cela me semble indiquer 
une forte affinité personnelle avec le travail de Delsarte.

L'approche de Clóvis Massa, par son association profonde de la théorie et de la pratique (ce qui est en cohérence avec la dynamique delsartienne) et par son ancrage dans le monde du théâtre, est venue compléter ma propre approche. Celle-ci a été plus inspirée par le monde de la danse et de l'éducation somatique. Et si elle ambitionnait également d'intégrer le système (théorique) à la méthode (pratique) de Delsarte (impliquant une expérimentation personnelle, devenue la base d'une transmission vivante depuis 2009), elle s'est néanmoins faite dans un cadre tout différent, celui d'un doctorat d'histoire dans lequel la pratique a été une option, un choix pédagogique : les illustrations par l'image (photographies et vidéos) ont d'abord été des aides pour rendre plus accessibles les textes décrivant des exercices. Dans une volonté de remonter au plus près des enseignements d'origine, j'ai élargi au maximum le champ des sources utilisées, adjoignant aux documents se trouvant dans les archives de Delsarte des deux côtés de l'Atlantique, les publications de ses élèves (Delaumosne, 1874 ; Arnaud, 1882 ; Giraudet, 1895 ; Hamel, 1906) et l'ouvrage de Stebbins (1977) qui fait référence, ainsi que les documents des archives américaines des élèves de Delsarte, Steele Mackaye (PMF) et Thomas-Étienne Hamel (FH), et de Henrietta Hovey (PRH \& TSC), élève d'un fils de Delsarte (Gustave).

Le visionnage de la démonstration de Clóvis Massa devant son jury m'a permis de repérer immédiatement des éléments d'expressivité venant, soit ajouter des données à ce que j'avais déjà proposé, soit préciser des points. Le travail de Massa m'a apporté en particulier deux éléments. D'abord, il m'a amené à indiquer plus nettement l'initiation par le thorax de certaines attitudes (la troisième, et surtout la cinquième, cf. infra), dans une dynamique moins « dansée » et plus proche de la vie quotidienne, ce qui est absolument l'esprit du travail des attitudes chez Delsarte, puisqu'elles viennent directement de ses observations de la vie de tous les jours. Ensuite, il m'a invité à une relecture des textes décrivant ces attitudes afin de mettre en relief la possibilité, pour certaines d'entre elles, de les faires avec les genoux droits ou pliés (c'est le cas pour toutes les attitudes appelées normales, c'est-à-dire dans lesquelles le poids est également réparti sur les deux pieds).

Je propose les résultats de cette approche combinée de ma propre recherche avec celle de Clóvis Massa par des photographies auxquelles j'ajoute mes commentaires. Je ne redonne pas l'ensemble des textes et images permettant ces interprétations afin de ne pas alourdir cet article, sachant que ces matériaux ont déjà été largement présentés par le passé dans mes publications.

Figure 3 : Première attitude de base (interprétation par l'image)

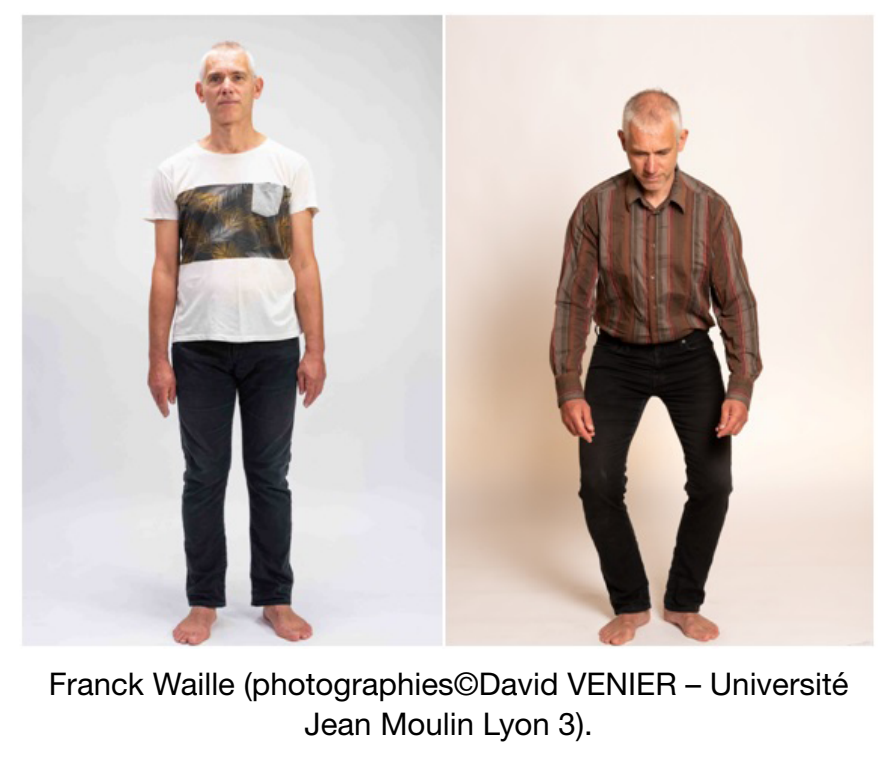

Cette attitude (Figure 3) « consiste dans 
une égale répartition du poids du corps sur les deux jambes » (Mackaye, 1869/1870b, p. 1). La double position des jambes est possible dans la mesure ou il n'y a aucune indication particulière sur ce point-là (ce qui laisse les deux possibilités ouvertes), et que cela correspond bien aux variétés d'expressions qu'évoque Delsarte. Ainsi, les jambes tendues renvoient plutôt à l'attitude « du soldat au port d'armes - moins la raideur qu'affecte une régularité voulue par une discipline inflexible » ou à celle «que prend l'homme dans le salut exprimant le respect » (ibid.). Les jambes pliées, elles, me semblent pouvoir être plus en lien avec « la faiblesse de l'enfance ou de la caducité » (ibid.) ou avec la position « de l'enfant qu'on met pour la première fois sur ses jambes 》(Delsarte, 1839 , p. 24). Cette photographie d'une petite fille se baissant donne une belle illustration de l'utilisation de cette attitude (en version un peu élargie) par un enfant (Figure 4) :

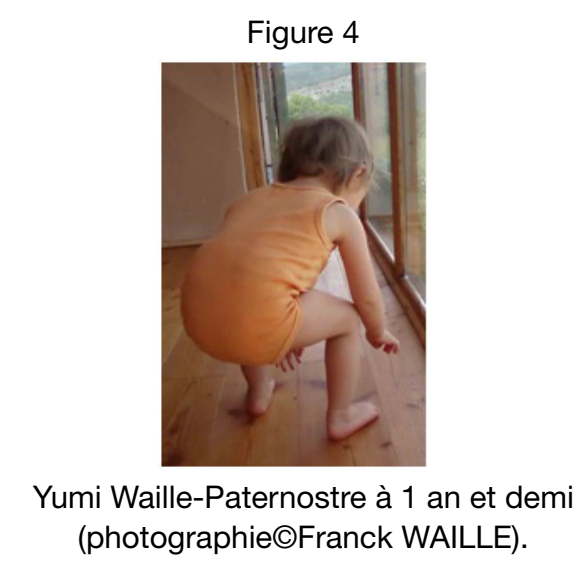

Cette égale répartition du poids sur les deux pieds génère l'équilibre du corps de manière plutôt statique. Chez Delsarte, les positions d'équilibre ne sont jamais conçues comme des arrêts absolus, mais comme des moments d'un déplacement, qui sont favorisés par la position non bloquée, libre, « déverrouillée » des genoux (cf. Waille, 2016, chapitre 7). Le genou libre permet de passer sans rupture de l'équilibre sur deux jambes à un déséquilibre qui va se résoudre par un nouvel équilibre, comme cela se passe par exemple quand sont enchaînées la première et la deuxième attitude (Figure $5)$.

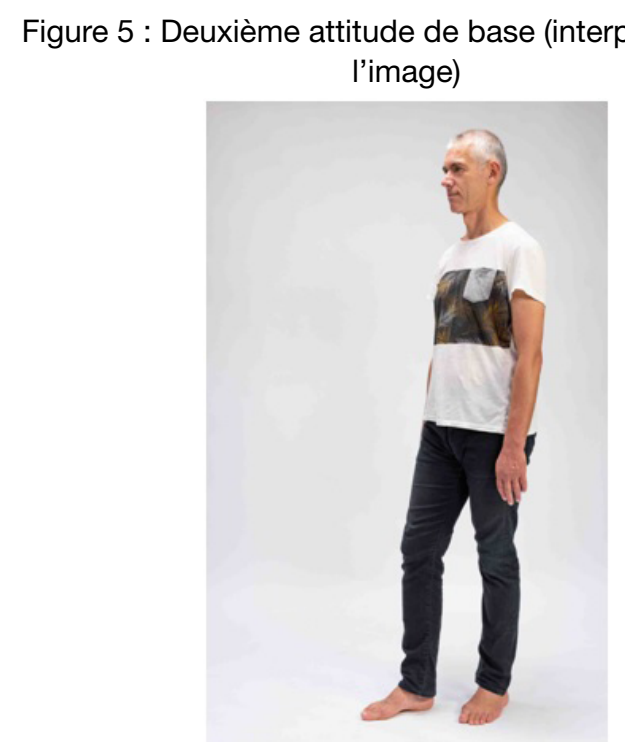

Franck Waille (photographies@David VENIER - Université Jean Moulin Lyon 3).

La deuxième attitude de base est concentrique : le poids est entièrement sur la jambe arrière, " on y passe en laissant tomber le poids du corps sur l'une ou l'autre hanche et en emportant la jambe qui se trouve libre en avant (...) sans tension ni raideur » (Mackaye, 1869/1870b, p. 1). Cette attitude caractérise selon Delsarte « le repos dans la force » (...) « certaines passions concentriques qui se cachent sous le dehors du calme » (ibid.), « la force, le calme, l'indépendance » (Delsarte, 1839, p. 24-25). La fameuse photographie de Victor Hugo contemplant la mer sur la grève d'Azette, la jambe gauche tendue et la jambe droite relâchée, est une belle illustration de cette attitude de repos observée dans la vie quotidienne (même si Hugo verrouille le genou de sa jambe d'appui). Nous en proposons un 
schéma au crayon (Figure 6) :

Figure 6 : Victor Hugo sur la grève d'Azette

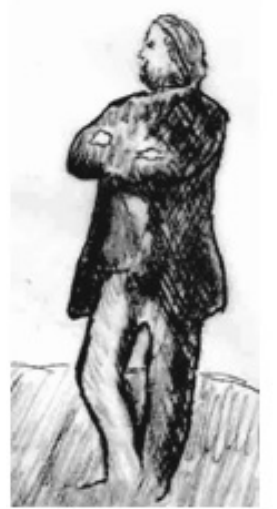

Dessin d'après la photographie de Charles Hugo (1852@ Franck WAILLE)

La manière qu'a Delsarte de décrire la transition entre la première et la deuxième attitude indique la qualité qu'il recherche dans le mouvement : «sans tension ni raideur ». L'indication de «laisser tomber le poids du corps sur l'une ou l'autre hanche » (et non de " porter le poids») renforce cette idée que c'est dans le relâchement que se fait le mouvement. Que ce soit la hanche qui est ici mentionnée (et non la jambe) est une manière d'insister sur l'idée de sensation de repos associée à cette attitude, parce que ce n'est pas l'action de soutenir le corps (action de la jambe d'appui) qui est mise en avant, mais celle de le recevoir (le poids du corps repose, d'une certaine façon, dans la hanche). De plus, mettre l'accent sur la hanche et non sur la jambe focalise l'attention sur le tronc et les sensations proprioceptives.

Cette attitude permet de faire une remarque générale lors des déplacements induits (c'està-dire le changement de jambe forte) durant le travail de ces attitudes, remarque renvoyant à la relaxation (décomposition) dans le mouvement. Giraudet transmet en effet que « si la jambe libre se retire directement (...), le mouvement a une gaucherie et une lourdeur inévi- tables, car le pied étant à plat [dans l'attitude de départ] pour retourner à plat dans l'attitude [d'arrivée], il y a un déplacement de la jambe sans désarticulation du tarse et du genou ; de là une masse mue lourde et disgracieuse " (1895, p. 120). II s'agit donc de veiller au relâchement des articulations de la jambe en mouvement, en particulier au niveau de la cheville (mais aussi du genou). Nous retrouvons là les indications de qualité («sans tension ni raideur ») données pour le passage de la première à la deuxième attitude, et l'inclusion de la décomposition dans le mouvement expressif comme « élément de respiration ».

\section{Figure 7 : Troisième attitude de base (interprétation par} l'image)

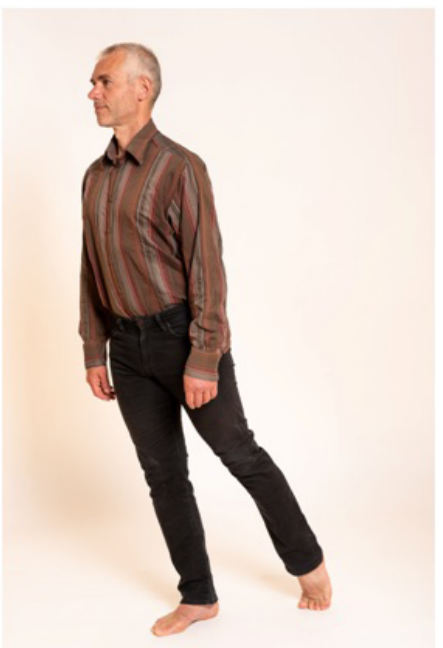

Franck Waille (photographies@David VENIER - Université Jean Moulin Lyon 3).

Cette attitude (Figure 7) « consiste à porter tout le poids du corps en avant et à tendre la jambe de derrière dans des proportions égales au degré d'avancement du torse " (Mackaye, 1869/1870b, p. 3). Elle « caractérise la véhémence dont elle est le type. Elle est l'attitude excentrique par excellence » (ibid.), « elle caractérise les sensations de la colère » (Van Hymbeck, 1843, chapitre 2).

Le texte indique sans ambiguïté quel est le 
moteur du mouvement : le torse, qui s'avance. Par torse, il convient bien de comprendre le haut du torse, c'est-à-dire la région du thorax.

Selon Delaumosne, cette attitude est particulièrement intéressante pour le chant : «on obtient une voix plus forte en prenant son point d'appui (...) à peu près sur la pointe des pieds, en prenant l'attitude de base que nous désignerons $(. .$.$) sous le terme de troisième "(1874, p$. 19) écrit-il. Ajoutons que des oppositions dans le corps (par exemple une opposition controlatérale associée à la troisième attitude des jambes) renforcent cette assise corporelle de la voix. II est possible d'en faire le constat en disant ou en chantant une même phrase dans différentes attitudes et organisations de l'instrument corporel, et de constater les modifications induites en termes de qualité et de puissance de l'émission. Précisons que la puissance vocale dont il s'agit ici n'a rien à voir avec une puissance forcée, c'est une puissance soutenue par l'organisme et son organisation.

Figure 8 : Quatrième attitude de base (interprétation par l'image)

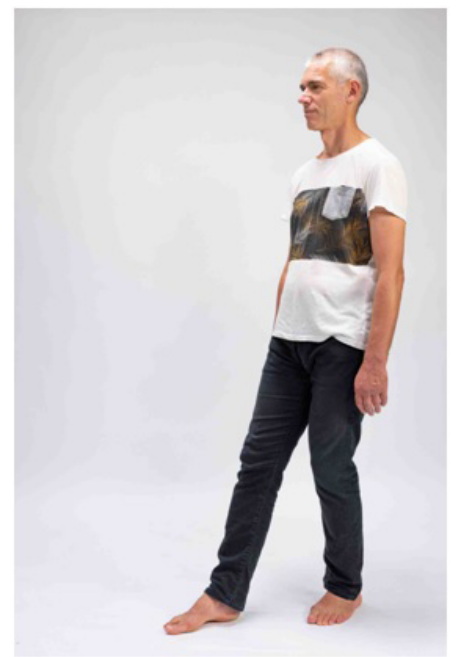

Franck Waille (photographies@David VENIER - Université Jean Moulin Lyon 3).

Cette attitude consiste à porter tout le poids du corps en arrière contrairement à l'attitude précédente où le corps est porté en avant, et à fléchir la jambe qui reçoit le poids du corps contrairement à l'autre qui doit être tendue (...) On peut du reste vérifier la régularité de cette attitude par l'agenouillement qui en est le paroxysme : si elle est bien faite, elle y conduit facilement (Mackaye, 1869/1870b, p. 3).

Elle « caractérise la faiblesse qui succède à la véhémence, elle est le type de la concentration, elle est aussi dans son caractère comme dans son aspect l'antipode de la troisième puisqu'elle en est l'expression réactive » (Mackaye, 1869/1870b, p. 3) (Figure 8); autrement dit, elle est associé à « l'accablement résultant de la véhémence spontanée des mouvements excentriques. La faiblesse naturelle se manifeste en 1re, la faiblesse soudaine en $4^{\mathrm{e}}$ » (Delsarte, 1839, p. 25).

Figure 9 : Cinquième attitude de base (interprétation par l'image)

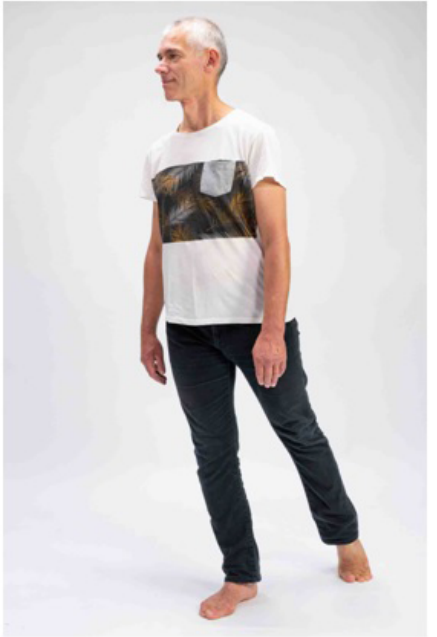

Franck Waille (photographie@David VENIER - Université Jean Moulin Lyon 3).

Cette attitude (Figure 9) « est nécessitée par le penchement du torse d'un côté ou de l'autre. C'est une espèce de $3^{e}$. C'est une des attitudes le plus importantes dans la tenue : c'est l'attitude transitive ou de passage, celle qui termine tous les angles opérés par la marche » (Delsarte, 1839, p. 25). Elle « sert de préparation aux marches obliques, elle est aussi incolore, 
transitive, suspensive, elle termine dans les marches tous les sommets d'angles » (Mackaye, 1869/1870b, p. 3).

Delsarte insiste pour bien faire démarrer le mouvement par le torse, qui entraîne le mouvement d'adaptation des jambes sur le côté. Cette attitude est essentiellement dynamique - d'où son aspect excentrique - car associée aux marches dans une diagonale : Laban (2003, p. 169) indique que la diagonale est la ligne la plus mobile (alors que si l'on va seulement sur le côté, il y a peu de mobilité). Dans la transmission faite par Mackaye, cette attitude perd son aspect dynamique car elle n'est pas associée à la marche sur une diagonale (1886, p. 52) ; elle possède alors un caractère statique. Cette déperdition d'information est d'autant plus dommageable que Delsarte affirme à propos de la $5^{\mathrm{e}}$ attitude que « c'est après la 2de l'attitude dont l'emploi est le plus fréquent »(1839, p. 25).

Dans ma version de 2009 (Figure 10), ma compréhension de la notion de « penchement du torse » était quelque peu raide :

Figure 10 : Ancienne version de la $5^{\mathrm{e}}$ attitude des jambes (2009)

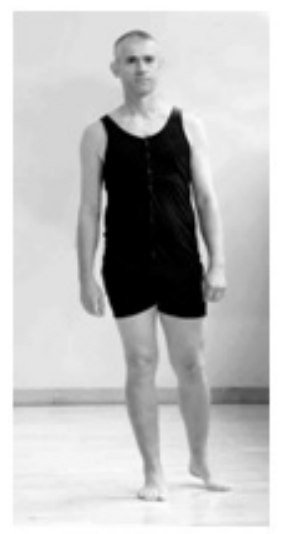

Franck Waille (photographie@David VENIER - Université Jean Moulin Lyon 3).

La version qu'en donne Clóvis Massa dans le film que j'ai visionné me semble beaucoup plus juste : le penchement du torse vue comme une orientation du torse dans une direction et le torse étant l'élément moteur, il se penche alors légèrement dans cette direction pour entrainer le reste du corps.

Figure 11 : Sixième attitude de base (interprétation par l'image)

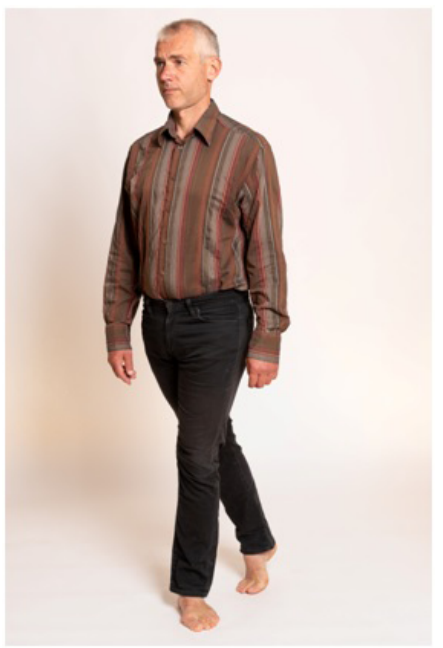

Franck Waille (photographies@David VENIER - Université Jean Moulin Lyon 3).

" La 6 e détermine des marches obliques, c'est une sorte de $3^{e}$ renversée » (Delsarte, 1839, p. 25) (Figure 11), « la pointe du pied de devant doit être très en dehors pour maintenir l'équilibre, l'on doit, pour vérifier l'exactitude de cette position, lever la jambe arrière " (Van Hymbeck, 1843, chapitre 2) : « la jambe libre de la cinquième devient (en se portant latéralement et légèrement en avant et fortement en dedans) la jambe forte et opère par cela un croisement avec la jambe de derrière " (Mackaye, 1869/1870b, p. 3).

C'est sans doute parce qu'elle est définie comme « une attitude de pompe, de cérémonie » (ibid.) qu'elle a été taxée d'avoir « quelque chose d'incompréhensible » par Giraudet (1895, p. 87, note 2), pour qui l'idée de respect et de révérence « ne peut s'accommoder avec les attitudes » : « cette manière de salut pom- 
peux ne pourrait entrer que dans la dynamique, et encore n'a-t-elle aucun caractère typique » (id., p. 87) ajoute-t-il. Giraudet a pris la responsabilité d'un changement, tout comme Mackaye et à sa suite les delsartistes américains. Mackaye remplace en effet cette attitude par une variante de la troisième attitude avec la jambe forte pliée (qu'il appelle $8^{\mathrm{e}}$ attitude des pieds). En voici une illustration (Figure 12), publiée par Stebbins :

Figure 12 : Attitude « excentric-excentric » des jambes selon Stebbins (1977, p. 153).

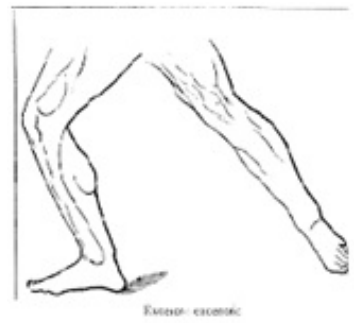

Dans un texte décrivant la neuvième attitude, Delsarte fait référence à « la quatrième qui est la seule attitude dont la jambe forte soit fléchie » (Mackaye, 1869/1870b, p. 4) : cette version américaine est donc en flagrante contradiction avec l'énoncé de Delsarte.

Hamel donne la clé pour expliquer la sixième attitude de base :

Enfin, la forme normale du genre excentrique (...) consiste en ce que la jambe forte est légèrement en avant de la jambe faible. C'est une position purement de transition, qui exprime la curiosité, l'inquiétude, et qui se résout promptement en une autre plus stable. Aussi Delsarte, s'écartant de sa rigueur théorique, l'avait-il remplacée par une autre qui est plutôt une marche révérencieuse qu'une attitude (1906, p. 155-156).

Cette attitude est donc essentiellement transitive et dynamique, elle permet le passage d'une attitude à une autre. II est significatif que Delsarte inclut précisément des attitudes «transitives » ou « de passage » au sein des attitudes de base. Cela illustre la place centrale chez lui du mouvement (ici compris comme déplacement dans l'espace) d'un point de vue pratique (tout comme théorique, cf. Waille, 2016, chapitre 3). Ainsi le mouvement a bien un « caractère typique » pour Delsarte, rendu par les $5^{\mathrm{e}}$ et $6^{\mathrm{e}}$ attitudes.

Dans les transmissions américaines, il est significatif que les deux attitudes de transition (la $5^{\mathrm{e}}$ et la $6^{\mathrm{e}}$ ) aient été soit supprimées, soit modifiées dans un sens statique. Plus généralement, si Mackaye reprend l'organisation générale en neuf attitudes des jambes (qu'il appelle " expressions des pieds avec l'équilibre harmonique » [ expression of feet with harmonic poise » $\left(1886,8^{\mathrm{e}}\right.$ leçon)]), il en supprime une, en introduit une nouvelle, en modifie partiellement d'autres et change la plupart de leur numérotation (cf. Waille, 2016, p. 359, note 272) : il présente d'abord trois attitudes normales, puis trois attitudes concentriques, et enfin trois attitudes excentriques (il n'utilise pas ce vocabulaire, qu'il a fini par évacuer de son enseignement - cf. Waille, 2017). Son classement montre que sa priorité n'est pas l'enchaînement des attitudes comme chez Delsarte, mais l'établissement d'une série de positions statiques. II y a alors un écart vis-à-vis de la démarche originelle établissant un lien entre les observations de la vie quotidienne et le travail expressif. Cela, associé à l'évacuation de l'aspect dynamique attaché à la $5^{\mathrm{e}}$ attitude et à la suppression de la $6^{\mathrm{e}}$ attitude, invite à penser que les modifications apportées par Mackaye concernant les attitudes des jambes pourraient en grande partie expliquer que le « système Delsarte-Mackaye »(Ruyter, 1991, p. 35) ait pu être perçu en Amérique comme essentiel- 
lement statique, et qu'il ait dérivé parfois vers des pratiques purement gymniques, deux caractéristiques étrangères au travail de Delsarte lui-même. Et si Stebbins - qui suit pourtant l'essentiel des modifications des attitudes des jambes apportées par Mackaye - est supposée avoir « ajouté la dynamique aux évolutions essentiellement statiques du système Delsarte-Mackaye " (ibid.), il faut bien comprendre cela dans le cadre du delsartisme et non de l'enseignement de Delsarte lui-même. Celui-ci est précisément orienté vers la dynamique de manière générale, et sur ce point particulier du travail des attitudes des jambes. La dynamique est au cœur même de l'art et de la pédagogie que Delsarte défend (cf. Waille, 2011).

Figure 13 : Septième attitude de base (interprétation par l'image)

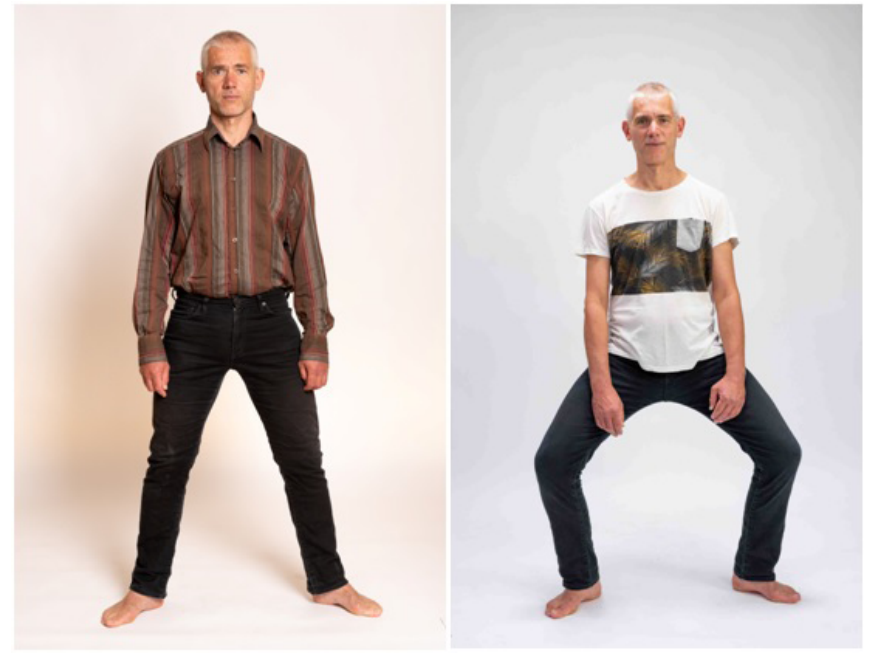

Franck Waille (photographies@David VENIER - Université Jean Moulin Lyon 3).

" Cette attitude est tout simplement une première écartée, et pour cette raison elle doit avoir de grandes affinités morales avec cette attitude. Cette attitude consiste dans la répartition du poids du corps sur les deux jambes parallèlement et fortement écartées 》 (Mackaye, 1869/1870b, p. 3-4) (Figure 13). Comme pour la première, il y a possibilité d'avoir les genoux soit droits, soit pliés (comme par exemple dans les arts martiaux orientaux). Elle est désignée par Delsarte comme celle du « repos absolu » (1859, cour pratique no 8 ) ; elle assure une grande stabilité ; elle « caractérise le responsable, c'està-dire l'attitude la plus forte, et par conséquent celle que prend l'homme frappé d'un coup mortel, en un mot elle est l'attitude du vertige ou de l'extrême confiance. Ne soyons donc pas étonnés du rapprochement de ces termes, si différents et si opposés, dans cette attitude, car il nous suffira de comprendre ce principe - que l'attitude forte est recherchée par la faiblesse comme l'attitude faible est affectée par la force » (Mackaye, 1869/1870b, p. 3-4).

Rappelons, concernant la notion de genoux droits, qu'il est central pour Delsarte de veiller à ce que les genoux soient toujours détendus, c'est-à-dire qu'ils soient toujours déverrouillés, autrement dit toujours potentiellement propres à assurer la mobilité dans l'espace. Les genoux sont donc droits sans être bloqués.

Figure 14 : Huitième attitude de base (interprétation par l'image)

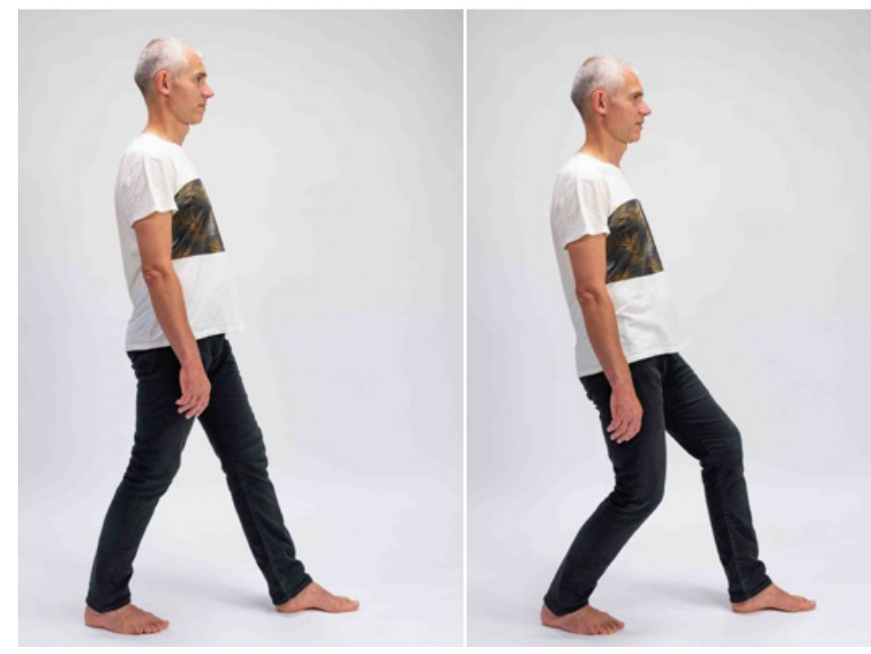

Franck Waille (photographies@David VENIER - Université Jean Moulin Lyon 3).

Voilà la dernière attitude normale (poids réparti sur les deux jambes) (Figure 14). « De 
septième, l'on doit par un mouvement de côté du torse faire pivoter sur la pointe ses pieds sans les bouger de place, de manière à avoir les deux pieds parallèles à la position du corps » (Van Hymbeck, 1843, chapitre 2). Delsarte la désigne comme « une attitude de parade, (...) également offensive et défensive » (ibid.) ; « elle sert pour l'excentricité avec réticence, pour la menace, pour la jalousie » (Delsarte, 1839, p. 25) ; « elle est le milieu exact entre la troisième et la quatrième, aussi exprime-t-elle l'alternative, au moral comme au physique, entre ces deux attitudes. L'homme placé entre la défensive et l'offensive prend toujours cette attitude alternative pour sonder les ressources de son courage en présence d'un ennemi plus fort que lui, il peut alternativement dans cette attitude avancer ou reculer » (Mackaye, 1869/1870b, p. 4).

Comme les autres attitudes normales, elle peut être faite avec les genoux droits ou pliés. En voyant le film de la démonstration de Clóvis Massa, j'ai trouvé que la version avec les genoux pliés était particulièrement parlante, par exemple dans le contexte d'une personne sur la défensive/offensive en arrivant dans un lieu potentiellement menaçant (mettant en garde en braquant son arme, par exemple, ou se préparant à pouvoir bouger dans n'importe quelle direction, ou en position de défense dans un art martial). J'ai tenté d'en proposer une illustration photographique ainsi :
Figure 15 : Variations possibles de la $8^{\mathrm{e}}$ attitude des jambes (jambes pliées) incluant les bras

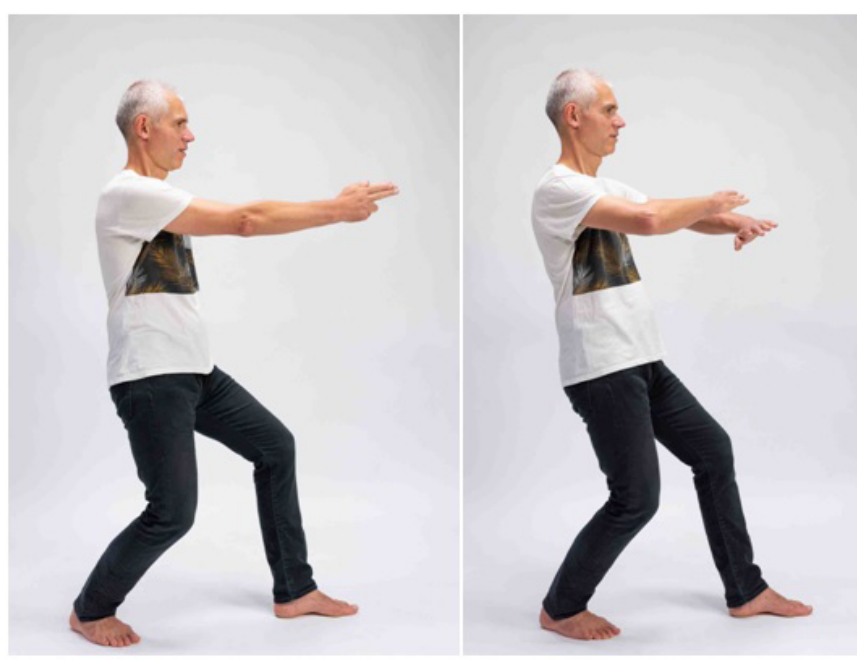

Franck Waille (photographies@David VENIER - Université Jean Moulin Lyon 3).

Dans les descriptions de cette attitude (Figure 15), le torse est encore très clairement désigné comme l'élément moteur par excellence. II serait en effet possible de passer de la septième à la huitième attitude par un pivotement des jambes ou par un mouvement du bassin, mais le manuscrit précise bien que c'est « par un mouvement de côté du torse » que les pieds et le corps dans son ensemble vont changer de position.

Par ailleurs, Delsarte donne une indication dynamique montrant comment il est possible de faire un exercice à partir de cette huitième attitude : « si l'on rompt l'on est en $4^{\mathrm{e}}$, si l'on avance l'on est en $3 e$ » (Van Hymbeck, 1843, chapitre 2). Autrement dit, il propose d'expérimenter, jambes écartées sur une ligne avant/ arrière, le passage d'une égale répartition du poids sur les deux jambes (huitième attitude), à celle du poids sur la jambe arrière (quatrième attitude) puis sur la jambe avant (troisième attitude). L'intérêt de cet exercice est de sentir, à partir d'une position équilibrée, le transfert progressif du poids sur la jambe qui est avancée ou sur celle qui est reculée, c'est-à-dire 
de travailler de manière fine la proprioception consciente, en lien avec la sensation du poids. Ce type de travail peut se rencontrer aujourd'hui dans un cours de danse contemporaine, avec de nombreuses variations.

Figure 16 : Neuvième attitude de base (interprétation par l'image)

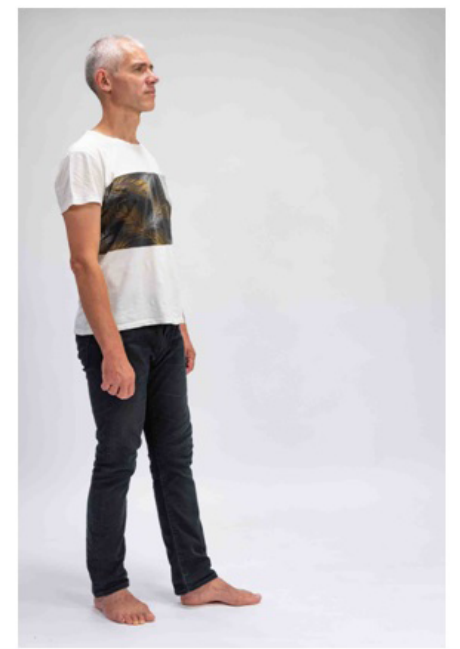

Franck Waille (photographie@David VENIER - Université Jean Moulin Lyon 3).

« Cette attitude est une deuxième tendue, elle diffère seulement en ce sens que la jambe libre est tendue au lieu d'être fléchie, comme dans la deuxième. (...) II faut (...) que le corps se porte avec force sur la hanche de la jambe forte afin que le côté de la jambe libre puisse s'allonger » (Mackaye, 1869/1870b, p. 4) (Figure 16). Elle « caractérise le défi » (ibid.). C'est « une attitude de parade ; elle est presque simultanément offensive et défensive. Son aspect impressionne facilement, elle jette l'auditeur dans l'alternative. Que va-t-il se passer? Quel sentiment va venir de cette l'attitude qui doit avoir sa résolution soit en $3^{\mathrm{e}}$, soit en $4^{\mathrm{e}}$ » (Delsarte, 1839, p. 25).

Cette dernière indication insiste sur le caractère dynamique de cette attitude, qui doit trouver à se résoudre de manière excentrique ( $3^{e}$ attitude) ou très concentrique (4e attitude).
Symboliquement, cette tendance de la neuvième attitude à se résoudre dans une autre attitude fait écho à la dynamique du chiffre 9 : fin du cycle de base (de 1 à 9), ouvrant sur le cycle suivant (10, ou retour à l'unité 1) (cf. Chevalier, Gheerbrant, 1982, p. 663-665).

Delsarte propose, comme le précise Giraudet, de s'entrainer à enchaîner les différentes attitudes du corps « de toutes les manières » $(1895$, p. 119), c'est-à-dire dans n'importe quel ordre. Pour les neuf attitudes des jambes, les manuscrits donnent des indications pour les enchaîner de la première à la neuvième, ou alors de les enchainer par groupes de trois (les normales, les excentriques, et les concentriques). Dans tous les cas, il est clair que c'est tant chacune des attitudes en elle-même qui importe pour Delsarte, que le mouvement de leur enchainement : nous sommes constamment dans le passage de la statique à la dynamique.

Ajoutons que Giraudet est le seul des élèves de Delsarte à présenter trois grands genres d'attitudes des jambes : le genre excentrique, quand la personne se tient debout ; le genre concentrique, quand la personne se tient à genoux ou a une fesse posée au sol ; et le genre normal quand la personne est assise (sur un support, et non directement sur le sol) (1895, p. 87-93).

Conclusion

Les attitudes des jambes sont bien les bases de la dynamique : elles sont la base de l'organisation corporelle générale. Les autres attitudes ayant une incidence directe sur l'organisation globale du corps sont celles du torse. Elles ont un lien direct avec la notion d'équilibre harmonique caractéristique des transmissions delsartiennes. Je présenterai ces éléments dans un second article à paraître à la suite de celui-ci. 
II importe de redire (comme dans mes publications de 2011 et de 2016) que les photographies sont ma compréhension et une partie de mon commentaire des informations recueillies. Elles n'ont ni caractère normatif ni prétention esthétique. Elles sont, par nature, susceptibles d'évoluer, et c'est le motif premier de cet article : proposer une mise à jour d'interprétations possibles de documents, mise à jour qui reste elle aussi susceptible d'évoluer. Les photographies présentées ici ont été faites spécialement pour cet article et présentent des différences notables par rapport à d'autres publiées précédemment sur le même sujet, nous en avons vu un exemple. Par ailleurs, la variété des informations transmises par différents élèves et/ou documents renvoie très certainement aux différentes variations et nuances que Delsarte introduisait dans son enseignement, variations qui invitent à cette dynamique ouverte et sans cesse susceptible de nouvelle interprétation. La rencontre avec l'approche de Clóvis Massa a mis en valeur la richesse de cette variété d'interprétations possibles.

Les photographies présentées ici ne prétendent donc rien être d'autre que de simples illustrations d'une interprétation, à un moment de ma recherche, des propositions d'entrainement auxquelles elles renvoient. Supports visuels de textes, elles comportent de nombreuses imperfections que le lecteur me pardonnera... et qu'il aura, je l'espère, le goût de gommer en s'impliquant dans la pratique des exercices exposés. Si cet article lui en donne l'envie, il aura atteint son but !

Abréviations

DC [= Delsarte Collection] : «François Alexan- dre Nicolas Delsarte Papers, Mss. 1301, Louisiana and Lower Mississippi Valley Collections, LSU Libraries, Baton Rouge, La. ».

FH : Fonds Hamel, Fonds des archives du Séminaire de Québec : SME (1626-1994), Musée de la civilisation, Service des collections, des archives historiques et de la bibliothèque, Québec (Québec), Canada.

MPF : The papers of the Mackaye Family, Baker Library, Special Collections, Dartmouth College, Hanover, New Hampshire, USA (Manuscript ML-5, Series 7, Steele MacKaye, 18481916, box 7 \& box 8 / Manuscript ML-5, Series 22, Addendum 1, box 219).

PRH : The Papers of Richard Hovey, Baker Library, Special collections, Dartmouth College, Hanover, New Hampshire, USA.

TSC : Ted Shawn Collection, Lincoln Center, Jerome Robbins Dance Division, The New York Public Library for the Performing Arts, New York, USA, cote : (S) *MGZMC-Res. 31, série VIII «Delsarte System of Expression ».

Bibliographie et sources (imprimées ou manuscrites)

ALEXANDER, Frederick Mathias. L'usage de soi. Bruxelles, Éditions Contredanse [The Use of the Self]. 1996 [1923].

ARISTOTE. Marche des animaux. Mouvements des animaux. Index des traités biologiques. Paris, Les Belles Lettres. 1973. 
ARNAUD, Angélique. François del Sarte, ses découvertes en esthétique, sa science, sa méthode, précédé de détails sur sa vie, sa famille, ses relations, son caractère. Paris, Ch. Delagrave. 1882. BAINBRIDGE-COHEN, Bonnie. Sentir, ressentir et agir. L'anatomie expérimentale du Body-Mind Centering $®$ (traduction de Sensing, feeling and action, par Madie Boucon). Bruxelles, Nouvelles de Danse $n^{\circ}$ 50. 2002.

BARBA, Eugenio. SAVARESE, Nicola. L'Énergie qui danse. Dictionnaire d'anthropologie théâtrale, Montpellier, L'Entretemps. 2008.

BARTHÉLÉMY, Yva. La voix libérée. Paris, Robert Laffont. 1984.

BERTHOZ, Alain. Le sens du mouvement. Paris, Odile Jacob. 1997.

BERTHOZ, Alain. La décision. Paris, Odile Jacob. 2003.

CABLE (s.d.), Notebook of Cable while studying with Delsarte [cahier de Cable étudiant avec Delsarte] (DC, box 2c, folder 132).

CHEVALIER, Jean; GHEERBRANT, Alain. Dictionnaire des symboles. Paris, Bouquins, Robert Laffont / Jupiter. 1982.

DAWN CLARK, Janis. The Influence of the Delsarte System of Expression on American Acting 1871-1970. Ph.D. Dissertation, Washington State University. 1982.

DELAUMOSNE, Abbé. Pratique de l'art oratoire de Delsarte. Paris, Joseph Albanel. 1874.

DELSARTE, François. École de Delsarte,
École de chant morale et scientifique. Notes et compte-rendu de ses cours, 1839 (DC, box 11b, folder sans \#). Différents transcripteurs, avec annotations et signature de Delsarte. 1839.

DELSARTE, François. Cours de M. Delsarte aux Sociétés savantes, cours $\mathrm{n}^{\circ} 8$ (DC, box 12 b, folder 54). 1858.

DELSARTE, François. Esthétique appliquée, cours de F. Delsarte. Exposition en neuf leçons de l'art de l'orateur, du peintre et du musicien. Offert à M. Delsarte par son élève Alphonse Pages (DC, box 12c, folder 40). 1859.

DELSARTE, François. Beau (DC, box 9-10, folder OS 36a, document 3). (s.d.).

FERNANDES, Ciane. The Moving Researcher : Laban/Bartenieff Movement Analysis in Performing Arts Education and Creative Arts Therapies. Kingsley, Jessica Publisher. 2014.

GIRAUDET, Alfred. Mimique, Physionomie et Gestes. Méthode Pratique D'après le système de François Del Sarte pour servir à l'expression des sentiments. Paris, Ancienne Maison Quantin / Librairies-Imprimeries Réunies. 1895.

GODARD, Hubert. «Le geste et sa perception.» In : GINOT, Isabelle; MICHEL, Marcelle. (Org.). La Danse au XX Siècle. Paris, Bordas, 1995. p. 224-229.

GUIDO, Laurent. L'Âge du rythme - Cinéma, musicalité et culture du corps dans les théories françaises des années 1910-1930. Lausanne, Éditions Payot/Jacques Scherrer éditeur. 2007.

HAMEL, Thomas-Étienne. Cours d'éloquence parlée d'après Delsarte. Québec, Imprimerie 
de la compagnie de l'Événement. 1906.

JOUSSE, Marcel. Étude de psychologie linguistique. Le style orale rythmique et mnémotechnique chez les verbomoteurs. Paris, Beauchesne, 1925.

LABAN, Rudolf. La maitrise du mouvement. Arles, Actes sud. 1997.

MACKAYE, James Steele. Notebook of Mackaye while studying with Delsarte \#3/7 [cahier de Mackaye étudiant avec Delsarte $n^{\circ} 3 / 7$ ] (DC, box 12b, folder 7). 1869/1870a.

MACKAYE, James Steele. Notebook of Mackaye while studying with Delsarte \#12 [cahier de Mackaye étudiant avec Delsarte $\left.n^{\circ} 12\right]$ (DC, range 35). 1869/1870b.

MACKAYE, James Steele. Harmonic Gymnastics (DC, box 6, folder 76). 1886.

MASSA, Clóvis Dias. A significação corporal a partir de François Delsarte. Projeto de Graduação, ligado à FAPERGS através da pesquisa teórica "Emprego de Signos Gestuais", Departamento de Arte Dramática da Universidade Federal do Rio Grande do Sul (UFRGS), Porto Alegre, 1991.

PORTE, Alain. François Delsarte, une anthologie. Paris, IPMC. 1992.

PRADIER, Jean-Marie. La scène et la fabrique des corps. Éthnoscénologie du spectacle vivant en Occident. Ve siècle avant Jésus-Christ$X V I I I{ }^{e}$ siècle. Talence, Presses Universitaires de Bordeaux. 2000.
RUYTER, Nancy Lee Chalfa. "Delsarte, son système et les États-Unis ", in François Delsarte, 1811-1871. Sources-Pensée. Châteauvallon, Théâtre National de la Danse et de I'Image, p. 33-37. 1991.

SMALLS, James. L'homosexualité dans l'art. New York, Parkstone Press. 2003.

STEBBINS, Genevieve. Delsarte System of Expression. New York, Dance Horizons (réédition de la sixième édition [1902] de l'ouvrage de Stebbins, initialement intitulé Delsarte System of Dramatic Expression, publié pour la première fois en 1885). 1977.

VAN HYMBECK, Auguste. Résumé des leçons de chant, grammaire, d'attitudes etc. du cours de M. Delsarte. Cahier de Van Hymbeck étudiant avec Delsarte (DC, box 8, folder 9, document 2). 1843.

WAILLE, Franck. Corps, arts et spiritualité chez François Delsarte (1811-1871). Des interactions dynamiques. Lille, ANRT, (version de soutenance [2009] en ligne, chapitre par chapitre, à l'adresse https://scd-resnum.univ-lyon3.fr/ out/theses/2009_out_waille_f.pdf). 2011.

WAILLE, Franck. "Les archives de François Delsarte, sources et produit d'un travail scientifique », in Waille, Damour (2015) : 19-22. 2015a.

WAILLE, Franck. « Retrouver la vitalité des enseignements de Delsarte : expressifs (ni stéréotypés, ni physiognomoniques) et inscrits dans le mouvement (non figés) ", in Waille, Damour (2015) : 77-94. 2015b. 
WAILLE, Franck. « Alfred Giraudet, artiste lyrique et pédagogue delsartien ", in Waille, Damour (2015) : 113-126. 2015c.

WAILLE, Franck. « Le travail corporel expressif de Delsarte : des observations du quotidien à un entraînement corporel structuré ", in Waille, Damour (2015) : 273-280. 2015d.

WAILLE, Franck. La méthode somatique expressive de François Delsarte. Historique, esthétique, anthropologique : de la neurophysiologique à la métaphysique. Montpellier, L'Entretemps. 2016.

WAILLE, Franck. « François Delsarte et les arts de la scène : l'enquête américaine ». Mis en ligne sur le site de l'Acfas (Association francophone pour le savoir), juin 2017 (http://www. acfas.ca/publications/decouvrir/2017/06/francois-delsarte-arts-scene-enquete-americaine). 2017.

WAILLE, Franck, DAMOUR, Christophe (dir.) François Delsarte, une recherche sans fin. Paris, L'Harmattan. 2015.

WINKIN, Yves. La Nouvelle Communication. Paris, Seuil. 2000.

(Je traduis en français les textes venus de l'anglais). 